\title{
The Early Growth of the First Black Holes
}

\author{
Jarrett L. Johnson ${ }^{1,3}$ and Francesco Haardt ${ }^{2}$ \\ ${ }^{1} \mathrm{X}$ Theoretical Design, Los Alamos National Laboratory, Los Alamos, NM 87545, USA \\ ${ }^{2}$ DiSAT, Universitá dell'Insubria, via Valleggio 11, 22100 Como, Italy \\ ${ }^{3}$ Email: jlj@lanl.gov
}

(ReCEIVED January 4, 2016; ACCEPTED January 30, 2016)

\begin{abstract}
With detections of quasars powered by increasingly massive black holes at increasingly early times in cosmic history over the past decade, there has been correspondingly rapid progress made on the theory of early black hole formation and growth. Here, we review the emerging picture of how the first massive black holes formed from the primordial gas and then grew to supermassive scales. We discuss the initial conditions for the formation of the progenitors of these seed black holes, the factors dictating the initial masses with which they form, and their initial stages of growth via accretion, which may occur at super-Eddington rates. Finally, we briefly discuss how these results connect to large-scale simulations of the growth of supermassive black holes in the first billion years after the Big Bang.
\end{abstract}

Keywords: accretion, accretion disks - radiation: dynamics - black hole physics - quasars: general - cosmology: theory

\section{INTRODUCTION}

Supermassive black holes (SMBHs) reside in the centres of massive galaxies (e.g. Kormendy \& Ho 2013), and the presence of quasars at the highest redshifts (e.g. Willott et al. 2003; Fan et al. 2006; Mortlock 2011; Venemans et al. 2013) powered by some of the most massive black holes (BHs) (e.g. Wu et al. 2015) suggests that this has been the case since the dawn of galaxy formation. It is a subject of intense research how these BHs initially formed and subsequently grew so rapidly (see previous reviews by e.g. Volonteri 2010, 2012; Volonteri \& Bellovary 2012; and Alexander \& Hickox 2012; Haiman 2013).

The formation of the first massive BHs begins with the collapse of the primordial gas to form their stellar progenitors. Depending on the environment in which these progenitors form, the mass scale of the seed BHs they leave behind can vary wildly, from $\sim 10$ to $\sim 10^{5} \mathrm{M}_{\odot}$. In turn, the rate at which these BHs initially grow is determined by their initial masses and the conditions of the gas reservoirs from which they accrete. While most BHs which form in the early universe are expected to grow at relatively modest rates, a small fraction may grow extremely rapidly, either due to their large initial masses and or to accretion flows which are heavy enough to stymie the radiation emitted in the accretion process. The final fates of the first massive BHs are dictated by the availability of gas to accrete on cosmological scales and by the impact of the merging and coalescence of these BHs with one another.
Here, we review recent theoretical exploration of these processes, from the formation of the first massive seed BHs, to the early stages of their growth and how they meet their fates as the supermassive BHs powering high-redshift quasars.

\section{INITIAL FORMATION OF THE FIRST MASSIVE BLACK HOLES}

We begin by discussing the factors determining the masses to which primordial stars grow prior to their collapse into the first massive BHs. We consider first the impact of the environment on the growth of the first stars. We then discuss the processes which determine their final masses.

\subsection{Impact of external sources of radiation}

The first stars form from the collapse of the primordial gas, a process which is facilitated by the radiative cooling of both atomic and molecular hydrogen $\left(\mathrm{H}_{2}\right)$ (e.g. Bromm \& Larson 2004; Greif 2015). Which of these two species of hydrogen dominates the cooling rate largely dictates the mode of star formation and the masses of the stars which form.

If molecular hydrogen forms in sufficient abundance, the primordial gas is expected to cool to $\sim 100 \mathrm{~K}$ and to collapse into protostars which accrete at rates of $10^{-4}-10^{-3} \mathrm{M}_{\odot} \mathrm{yr}^{-1}$ (e.g. Yoshida, Omukai, \& Hernquist 2008). At these accretion rates, the typical final masses of the stars which form are 


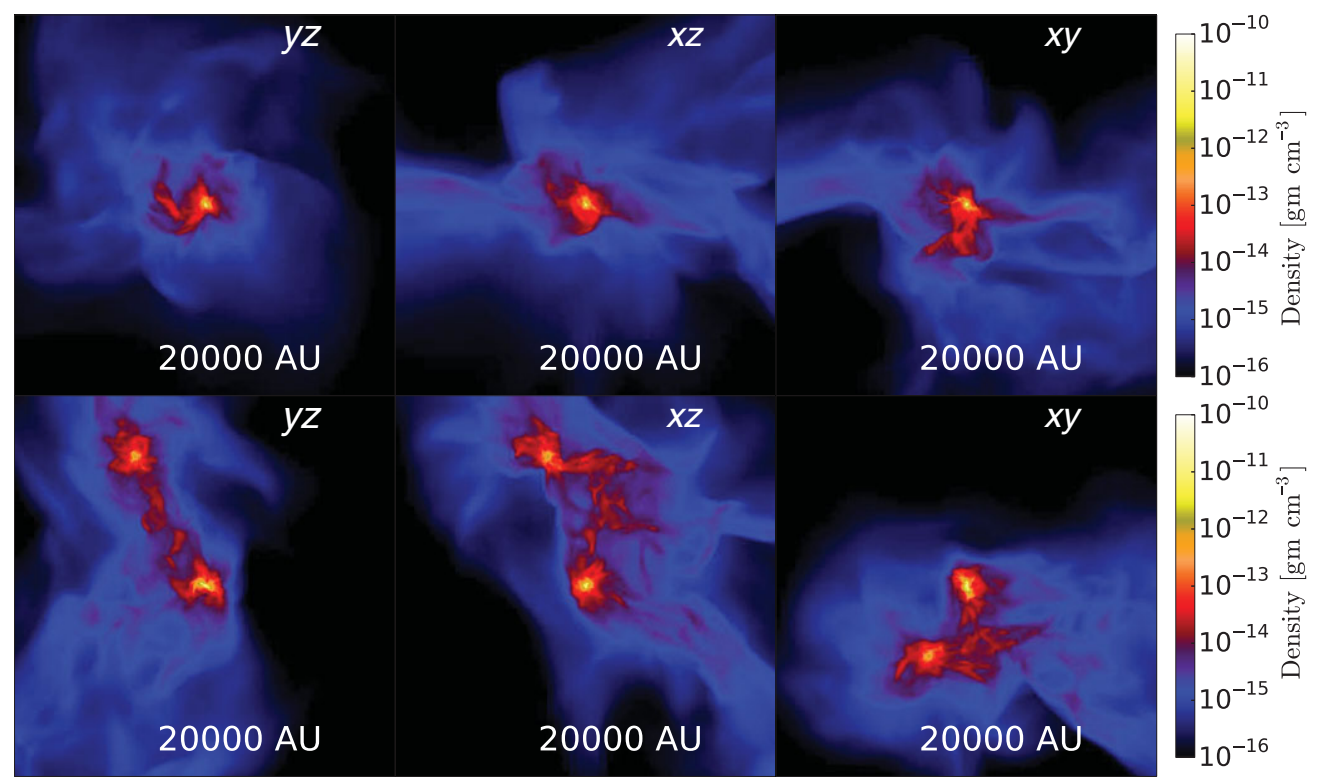

Figure 1. The projected gas density in halos hosting one (top row) and two (bottom row) rapidly growing primordial protostars, along the three axes in a cosmological simulation, as labelled. At the early stages of accretion shown here, the objects have masses a few times that of the Sun, but they are expected to grow rapidly to become $\geq 10^{4} \mathrm{M}_{\odot}$ supermassive stars within a few million years. From Latif, Schleicher, \& Hartwig (2015).

likely between $\sim 10$ and $\sim 10^{3} \mathrm{M}_{\odot}$ (e.g. Hirano et al. 2014). The outcome of the evolution of a large fraction of these stars is expected to be the formation of a $\mathrm{BH}$ with a mass approaching that of the star itself (e.g. Heger et al. 2003).

If by some means $\mathrm{H}_{2}$ formation is suppressed, then atomic hydrogen is left as the principle coolant. In this case, the gas cools only to $\sim 10^{4} \mathrm{~K}$ and is expected to collapse at extremely high rates of up to $\sim 1 \mathrm{M}_{\odot} \mathrm{yr}^{-1}$ into a supermassive star (SMS), as shown in Figure 1 (e.g. Koushiappas, Bullock, \& Dekel 2004; Lodato \& Natarajan 2006; Wise, Turk, \& Abel 2008; Choi, Shlosman, \& Begelman 2013, 2015). The typical final masses of such SMSs are expected to be $\sim 10^{5}$ $\mathrm{M}_{\odot}$, orders of magnitude larger than primordial stars formed from gas cooled by molecular hydrogen (e.g. Latif et al. 2013). The typical final outcome of the evolution of such massive objects is the formation of a $\mathrm{BH}$ with a mass which is a significant fraction of the SMS itself (e.g. Shibata \& Shapiro 2002; Begelman 2010; Ball et al. 2011; Fryer \& Heger 2011), although in some cases, it may be much lower due to winds that are generated during the collapse of the star (e.g. Dotan, Rossi, \& Shaviv 2011; Fiacconi \& Rossi 2016).

While it is possible that a variety of processes can lead to the suppression of gas cooling and, in turn, to the formation of SMSs (e.g. Sethi, Haiman, \& Pandey 2010; Inayoshi \& Omukai 2012; Tanaka \& Li 2014; Inayoshi, Visbal, \& Kashiyama 2015a), the process which is perhaps most likely to play a decisive role is the destruction of $\mathrm{H}_{2}$ molecules by intense ultraviolet radiation which is absorbed in the LymanWerner (LW) bands of the molecule (e.g. Haiman 2013; Fernandez et al. 2014; Visbal, Haiman, \& Bryan 2014a; Latif, Niemery, \& Schleicher 2014). For $\mathrm{H}_{2}$ cooling to be effec- tively suppressed, however, requires extraordinarily intense LW radiation (e.g. Bromm \& Loeb 2003). This suggests that SMS formation occurs only in relatively rare regions of the early universe, in pristine dark matter (DM) halos that are nearby galaxies which harbour stars producing sufficient LW radiation (e.g. Dijkstra et al. 2008, Dijkstra, Ferrara, \& Mesinger 2014; Agarwal et al. 2012; Visbal, Haiman, \& Bryan 2014b; but see Petri, Ferrara, \& Salvaterra 2012).

Estimates of the number density of SMSs, and of the seed BHs into which they collapse, are difficult to make, due to the sensitivity of the required level of LW radiation to the state of the collapsing gas (e.g. Wolcott-Green, Haiman, \& Bryan 2011; Hartwig et al. 2015a) and to uncertainties in the rates of key chemical reactions (e.g. Glover 2015). In addition, as $\mathrm{H}_{2}$ molecules typically form from $\mathrm{H}^{-}$and $\mathrm{H}_{2}{ }^{+}$ions, there is a strong sensitivity as well to the shape of the stellar spectrum producing the $\mathrm{LW}$ radiation, as these ions can be destroyed by lower senergy photons (e.g. Shang, Bryan, \& Haiman 2010; Sugimura, Omukai, \& Inoue 2014; Sugimura et al. 2015; Agarwal \& Khochfar 2015; Latif et al. 2015). Also, the same galaxies which produce LW radiation may also be sources of X-rays, which can penetrate deeply into collapsing halos and ionise the gas, leading to enhanced $\mathrm{H}_{2}$ formation rates (e.g. Machacek, Bryan, \& Abel 2003; Glover \& Brand 2003) and, in turn, to suppression of SMS formation (e.g. Inayoshi \& Omukai 2011; Inayoshi \& Tanaka 2015). Finally, even ionising radiation, at energies only slightly higher than LW radiation, can heat and ionise the gas at early phases in the collapse, leading to further suppression of SMS formation (e.g. Johnson et al. 2014; Yue et al. 2014; Regan, Johansson, $\&$ Wise 2015) 
It is worth noting that, while the impact of radiation on the prevalence of SMSs is complex, even moderate levels of $\mathrm{LW}$ radiation can lead to enhancements in the masses of primordial stars which collapse into seed BHs (e.g. O'Shea \& Norman 2007; Xu, Wise, \& Norman 2013; Latif et al. 2014; Hirano et al. 2015). Next, we turn to discuss other factors which determine the final masses of $\mathrm{BH}$ progenitors in the early universe.

\subsection{Expected mass scale of black hole progenitors}

The expected initial masses of the first seed BHs are determined largely by the masses to which their progenitor stars grow during their brief lifetimes. In turn, a number of processes can dictate how large these progenitors grow, depending on the environment in which they form.

The initial mass function of primordial stars formed from gas cooled by $\mathrm{H}_{2}$ in DM minihalos is likely set at the smallest mass scales by the ejection of sub-solar protostars by gravitational interactions, and at the largest scales by radiative feedback from the stars themselves which eventually halts their accretion of gas (see e.g. Bromm 2013; Greif 2015). Recent radiation hydrodynamics simulations with cosmological initial conditions suggest that the upper end of the initial mass function extends up to $\sim 10^{3} \mathrm{M} \odot$ (Susa, Hasegawa, \& Tominaga 2014; Hirano et al. 2014, 2015). Such massive primordial stars are expected to collapse to seed BHs with similar masses (e.g. Madau \& Rees 2001; Schneider et al. 2002; Heger et al. 2003; see also Islam, Taylor, \& Silk 2003).

If the first generations of stars form in tightly bound clusters, it is possible that mergers may drive the most massive stars to masses in excess of $10^{3} \mathrm{M}_{\odot}$ (e.g. Portegies Zwart et al. 2004; Devecchi \& Volonteri 2009; Davies, Miller, \& Bellovary 2011; Katz, Sijacki, \& Haehnelt 2015). This avenue of BH formation from slightly metal-enriched star clusters (e.g. Lupi et al. 2014) can also produce seeds which may subsequently grow to larger, supermassive scales (e.g. Devecchi et al. 2012; Yajima \& Khochfar 2016).

The expected mass scale of much more massive SMSs formed from accretion of atomically cooled primordial gas is likely set either by their limited lifetimes (e.g. Begelman 2010; Johnson et al. 2012) or by the depletion of the gas reservoirs from which they accrete (e.g. Schleicher et al. 2013). The radiative feedback from these objects is expected to be relatively weak, owing to the low temperatures of the bloated outer envelopes characteristic of such rapidly accreting objects (e.g. Hosokawa, Omukai, \& Yorke 2012, 2013; Begelman 2012). Even under intermittent high accretion rates, as expected due to accretion from a clumpy disk (e.g. Inayoshi \& Haiman 2014; Latif \& Volonteri 2015; Sakurai 2015a; Luo, Nagamine, \& Shlosman 2015), rapid gas infall appears to drive the expansion of the star episodically, limiting the emission of ionising photons, as shown in Figure 2 (Sakurai et al. 2015b). Likewise, high accretion rates are likely to be realised even in highly turbulent atomically cooled collapsing gas (e.g. Prieto, Jimenez, \& Haiman 2013; Van Borm

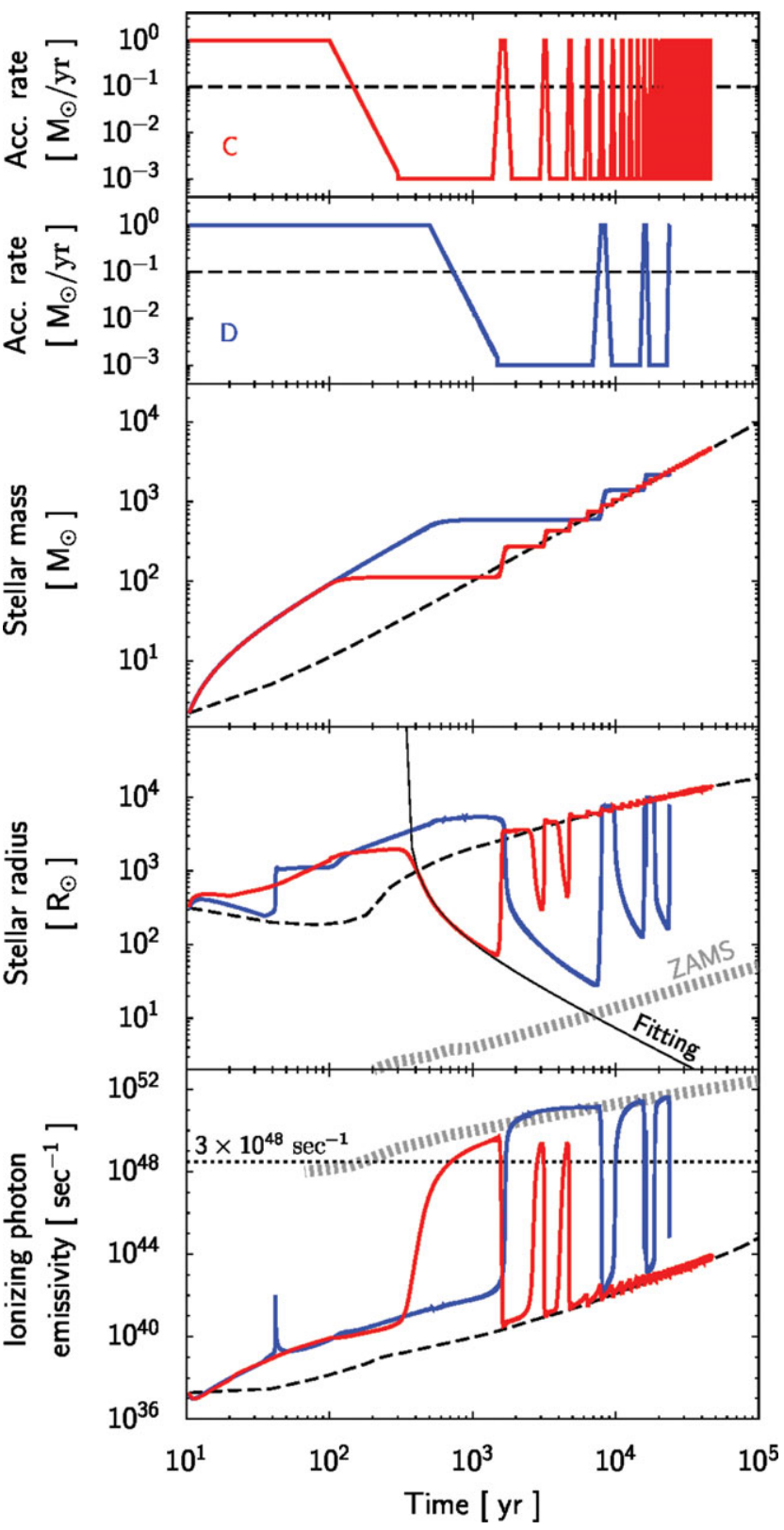

Figure 2. The properties of a growing supermassive star, as functions of time, for two different assigned time-varying accretion rates (shown by red and blue lines). During periods of slow accretion, the stellar radius shrinks, the emitting surface becomes hotter, and the rate of ionising photon emission increases. However, the impact of the ionising radiation on the accretion flow is not sufficient to stop the growth of the star. From Sakurai et al. (2015b).

et al. 2014). Once the conditions are met for the formation of a SMS, it thus appears there is little to limit its growth to a characteristic mass scale of at least $\sim 10^{5}$. The fate of most such massive objects is to collapse to a $\mathrm{BH}$ with a similarly large mass, although a large fraction of its mass may instead be lost if sufficiently large accretion rates persist up until its collapse (e.g. Begelman, Volonteri, \& Rees 2006; Dotan et al. 2011; Schleicher et al. 2013). 
An avenue for the formation of even more massive seed BHs, with initial masses up to $10^{8}-10^{9} \mathrm{M}_{\odot}$, has been suggested to be rapid gas inflow into the centre of rare, Milky Way-sized galaxies triggered by gas-rich mergers at high redshift (Mayer et al. 2010; Bonoli, Mayer, \& Callegari 2014; Mayer et al. 2015). While the nature of the initial conditions in this scenario is more complex than those based on the collapse of primordial gas in less massive DM halos, and may involve complications such as the pre-existence of BHs in the centres of the merging galaxies, somewhat idealised simulations suggest that heavy, optically thick accretion flows may well lead to the formation of such massive central objects (Mayer et al. 2015; see also Ferrara, Haardt, \& Salvaterra 2013).

\section{INITIAL GROWTH OF SEED BLACK HOLES}

The rate at which seed BHs grow, immediately following their formation, is dictated both by the mechanical and radiative feedback from their progenitor stars and by the impact of the radiation produced in the accretion process itself. Here, we discuss these processes and the basic expectations for the early growth of $\mathrm{BH}$ seeds.

\subsection{Impact of radiation emitted from the progenitor}

$\mathrm{BH}$ progenitors being massive stars with large luminosities, in many cases, the radiation they emit dramatically alters their surroundings. In turn, as it is from these surroundings that the nascent $\mathrm{BHs}$ accrete, the radiation from progenitor stars can play a key role in determining how quickly the first BHs grow.

Massive primordial stars formed from $\mathrm{H}_{2}$-cooled gas in minihalos are expected to produce copious amounts of ionising radiation for at least 1-2 Myr before their collapse (e.g. Schaerer 2002). The gas surrounding them is therefore heated to well above the virial temperature of their host DM halos, and a large, low-density H II region is left when the star collapses (e.g. Kitayama et al. 2004; Whalen, Abel, \& Norman 2004; Alvarez, Bromm, \& Shapiro 2006; Abel, Wise, \& Bryan 2007). Such BHs are thus expected to be born into very low-density environments, from which gas accretion is exceedingly slow, leading to a significant delay of perhaps up to $\sim 100$ Myr in the growth of these seeds (Johnson \& Bromm 2007; Alvarez, Wise, \& Abel 2009; Johnson et al. 2013).

In contrast, the radiative feedback from SMSs is likely to be much weaker, due to their cool surfaces from which little ionising radiation is emitted (e.g. Hosokawa et al. 2013; Schleicher et al. 2013; Sakurai 2015a; Sakurai et al. 2015b). This is likely to result in a large reservoir of dense gas from which such a so-called direct collapse black hole (DCBH) can accrete. Cosmological simulations of this process suggest that Eddington-limited accretion is possible starting from the formation of the DCBH (Johnson et al. 2011; Aykutalp et al. 2014), and more idealised models of the accretion flow at smaller scales near the DCBH suggest that even higher rates of growth are attainable (e.g. Pacucci \& Ferrara 2015; Inayoshi, Haiman, \& Ostriker 2015b). Thus, not only do SMSs produce large initial $\mathrm{BH}$ seeds, these seeds are also likely to grow rapidly starting from birth.

\subsection{Impact of progenitor explosion}

The supernovae ( $\mathrm{SNe}$ ) which mark the end of life of many massive primordial stars dramatically impact their surroundings, blowing out the gas from the centres of their host DM halos, thereby limiting the gas reservoir available for accretion onto any seed BHs that are left behind.

Primordial stars with masses of $140-260 \mathrm{M}_{\odot}$ are expected to explode as pair-instability SNe, with energies of $\sim 10^{53} \mathrm{erg}$ (e.g. Heger et al. 2003). Such energetic explosions can easily evacuate the gas from the centres of DM minihalos (e.g. Greif et al. 2007; Vasiliev, Vorobyov, \& Shchekinov 2008; Wise \& Abel 2008). While such SNe leave behind no BH remnant, it is likely that primordial stars in this environment form in clusters (e.g. Greif 2015) and such strong mechanical feedback can leave the $\mathrm{BH}$ remnants of other stars to accrete from low-density gas, resulting in very slow growth. Lower mass primordial stars may also explode as less energetic $\mathrm{SNe}$, which may or may not effectively evacuate their host minihalos of gas for long periods, depending on the energy of the explosion and on the mass of the minihalo (Kitayama \& Yoshida 2005; Whalen et al. 2008; and Ritter et al. 2012). Indeed, it is expected that a large fraction of primordial SNe may leave behind a remnant $\mathrm{BH}$, which may accrete gas that is not evacuated in the explosion, although some fraction of these BHs may be relegated to the low-density intergalactic medium (IGM) due to natal kicks imparted to them in assymetric SNe (Whalen \& Fryer 2012).

It is also a possibility that some SMS explode in extremely energetic SNe (e.g. Montero, Janka, \& Müller 2012; Chen et al. 2014). While these violent events may not leave behind a remnant $\mathrm{BH}$, it is possible that some SMSs form in binary systems (Regan \& Haehnelt 2009; Reisswig et al. 2013; Whalen et al. 2013c; Latif, Schleicher, \& Hartwig 2015; Becerra et al. 2015) and accretion onto a $\mathrm{BH}$ formed from the collapse of a companion SMS would likely be strongly inhibited due to the evacuation of high-density gas from the centre of the host halo (Johnson et al. 2013b; Whalen et al. 2013a,b). Finally, if a relativistic jet is launched from the centre of a collapsing SMS, perhaps powering a gamma-ray burst, it could also drive out the surrounding gas, resulting in a similar suppression of accretion onto the DCBH that is left behind (Matsumoto et al. 2015).

\subsection{Impact of radiation emitted in the accretion process}

The interaction between the accretion flow onto a $\mathrm{BH}$ and the radiation emitted in the accretion process is highly complex, and is sensitive to the initial conditions of the flow as well 


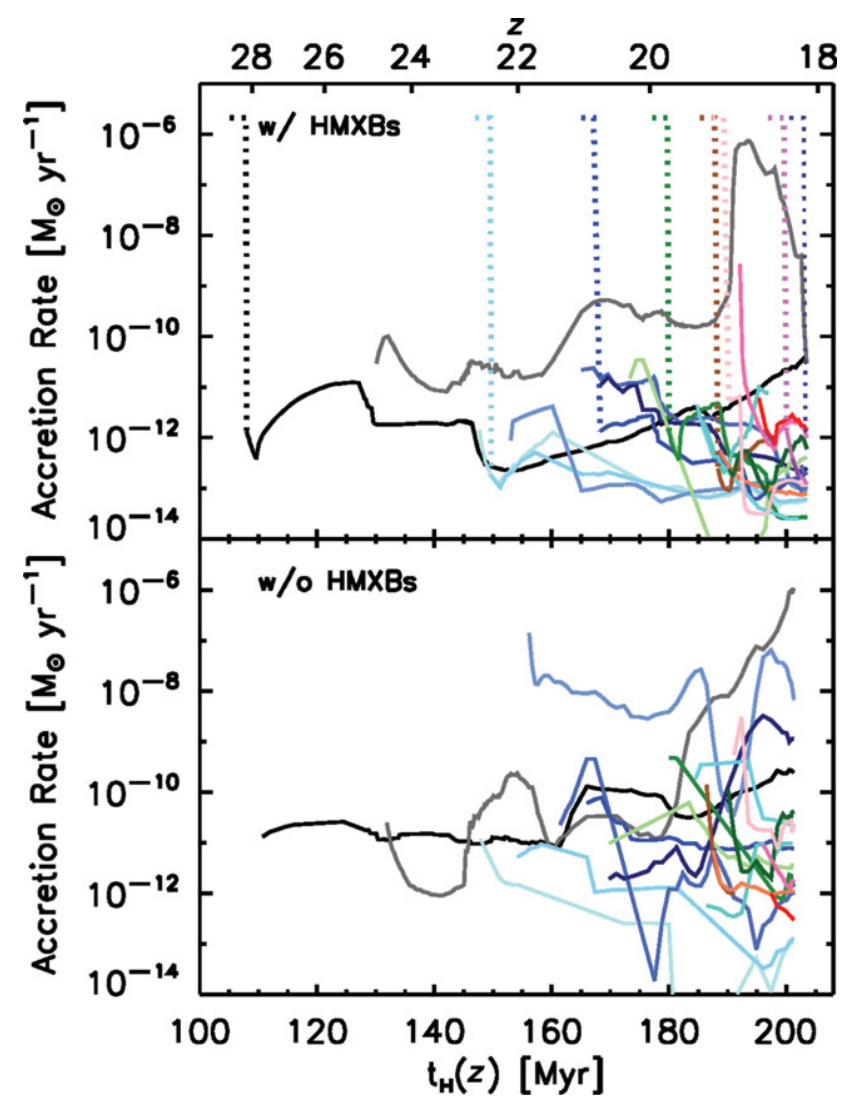

Figure 3. Accretion rates of BHs in simulations with (top) and without (below) radiative feedback from high-mass X-ray binaries. In conjunction with the radiative feedback from their stellar progenitors, the impact of this $\mathrm{X}$-ray feedback is to dramatically limit the accretion of gas onto BHs formed from primordial stars in minihalos. From Jeon et al. (2014).

as to physical effects on both small scales near the $\mathrm{BH}$ and larger scales where the flow originates.

In cases where a seed $\mathrm{BH}$ is accreting from a relatively lowdensity medium, such as occurs when the radiative and/or mechanical feedback from the progenitor effectively clears out the gas, the high-energy radiation that is generated in the accretion disk can travel large distances (e.g. Kuhlen \& Madau 2005), heating and expelling the gas. This can result in a dramatic suppression of accretion onto the $\mathrm{BH}$ seeds left by primordial stars formed in DM minihalos (e.g. Alvarez et al. 2009), and can be exacerbated if there is additional radiative feedback from X-ray binaries (e.g. Mirabel et al. 2011; Fragos et al. 2013; Jeon et al. 2014; Xu et al. 2014; Chen et al. 2015; Ryu, Tanaka, \& Perna 2015), as shown in Figure 3.

In cases where a seed $\mathrm{BH}$ is accreting from relatively dense gas, as is expected to occur for DCBHs born in dense regions little affected by feedback from the progenitor SMS, growth via accretion is much more rapid. Cosmological simulations resolving the Bondi radius of $\mathrm{DCBHs}$ and including ionising radiation (Johnson et al. 2011) and X-ray (Aykutalp et al. 2014) feedback, suggest that the accretion rate may at times peak near the Eddington rate, although it is on average well

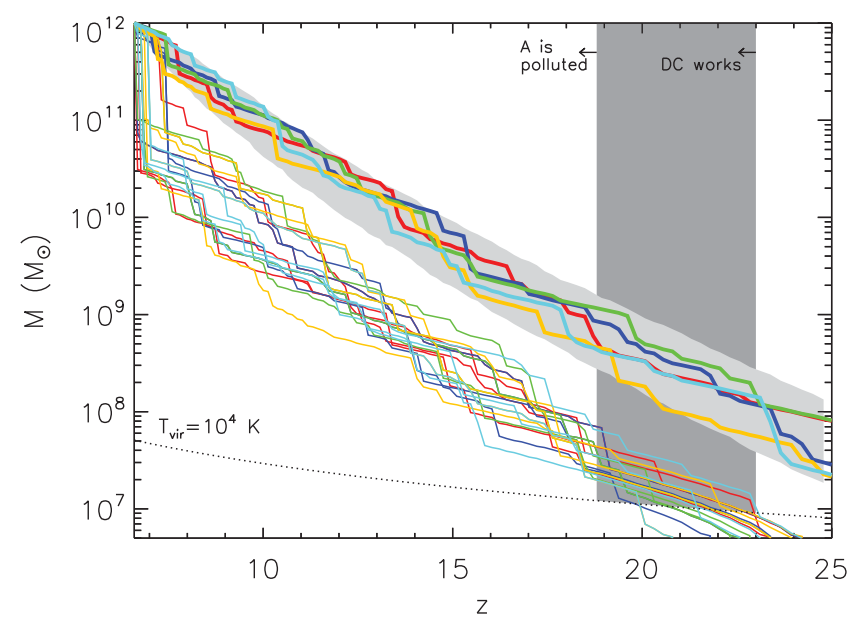

Figure 4. The distribution of possible masses of the DM halo hosting the candidate $\mathrm{DCBH}$ in the CR7 system (thin lines) and the corresponding masses of the larger DM halo with which it merges, as functions of redshift $z$. The mass of a DM halo with a virial temperature of $10^{4} \mathrm{~K}$ is denoted by the dotted line. In this scenario, the LW radiation emitted from stars formed in the larger halo destroys the $\mathrm{H}_{2}$ molecules in the smaller one until its virial temperature exceeds $10^{4} \mathrm{~K}$, at which point the gas collapses rapidly and forms a supermassive star that later collapses into a DCBH. From Agarwal et al. (2015).

below this value. Similar periodic behaviour is found in more idealised calculations which resolve smaller scales near the BH, as well (e.g. Milosavljević et al. 2009; Park \& Ricotti 2011, 2012). While this suggests that accretion onto DCBHs may occur at, on average, somewhat sub-Eddington rates, if accretion proceeds from sufficiently dense gas then radiation from the accretion disk can be stymied and the accretion rate may exceed the Eddington rate for long periods (e.g. Inayoshi et al. 2015; Pacucci, Volonteri, \& Ferrara 2015). Given the strong dependence of the final $\mathrm{BH}$ mass on the early accretion rate, it is clearly critical to determine the state of the environment in which a DCBH is formed in order to model its subsequent evolution and fate.

Intriguingly, the recently detected bright Lyman- $\alpha$ source in the CR7 system (Sobral et al. 2015) may provide strong empirical constraint on the feedback resulting from accretion onto DCBHs. Modelling of this source, in which no emission lines from heavy elements have been detected, suggests that it is likely to be powered by a DCBH accreting primoridial gas (e.g. Pallottini et al. 2015). This is consistent with an observed nearby galaxy which may have produced the LW radiation required for DCBH formation (Agarwal et al. 2015; see also Regan et al. 2015), as shown in Figure 4. This is also consistent with modelling that suggests that lower mass seed BHs formed in minihalos would not likely evolve into a system consistent with the data (Hartwig et al. 2015b), as shown in Figure 5. The lack of heavy elements inferred for this source implies that star formation has been suppressed continually for at least $\sim 10^{8} \mathrm{yr}$ (Pallottini et al. 2015) and perhaps longer (Agarwal et al. 2015). This may be explained by suppression of $\mathrm{H}_{2}$ formation due to the intense LW radiation emitted 


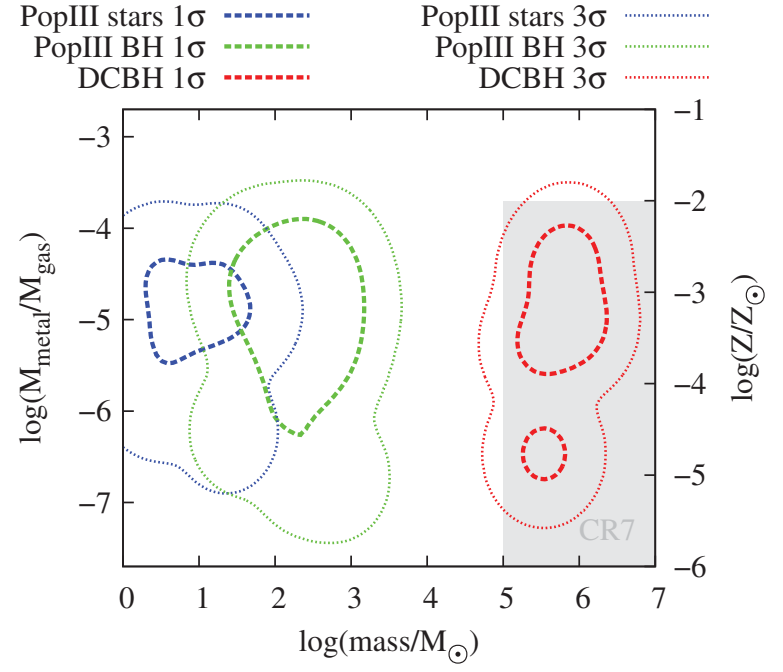

Figure 5. The metallicity of the bright Lyman- $\alpha$ emitter in CR7, as produced by modelling its emission as due to a cluster of primordial stars (blue), an accreting $\mathrm{BH}$ formed from the collapse of a primordial star in a minihalo (green), and an accreting DCBH (red), with the masses of these respective objects shown along the horizontal axis. The dashed and solid contours correspond to the range of properties found in 68 and $99 \%$, respectively, of the model realisations studied. The gray region is that in which the properties are consistent with CR7; only an accreting DCBH can simultaneously produce the bright emission observed while also suppressing metal enrichment strongly enough so as to limit the strength of metal emission lines to a level consistent with the observations. From Hartwig et al. (2015b).

from a DCBH accreting continually at a substantial fraction of the Eddington rate (e.g. Johnson et al. 2011) in conjunction with the strong LW radiation emitted from its neighbouring galaxies, although a countervailing effect is that the X-rays emitted from the accretion disk may promote molecule formation and star formation (Aykutalp et al. 2014), as shown in Figure 6. Cosmological simulations directed at modelling the formation of systems specficially like CR7 will help to shed more light on its nature.

\section{SUPER-EDDINGTON ACCRETION AS A PATH TO SUPERMASSIVE BLACK HOLES}

The need to ease the requirements set by $z \simeq 6$ QSOs was discussed, among others, by Volonteri \& Rees (2005), who envisaged an early phase of super-critical accretion in the growth of SMBHs. Within primordial halos with virial temperature $\gtrsim 10^{4} \mathrm{~K}$, in particular, conditions exist for the formation of a rotationally supported disk at the centre of the halo. The authors showed that if the disk rotates as a solid body, then a tiny accretion disk can form inside the radiation trapping radius, and hence the $\mathrm{BH}$ can accrete most of the infalling gas. On similar theoretical grounds, Kawakatu \& Wada (2009) proposed a simple model for the co-evolution of a clumpy circumnuclear disk and of a SMBH, and showed that the observed bright QSOs at $z \gtrsim 6$ are best explained by a late growth $(z \lesssim 10)$ of the $\mathrm{BH}$ seed at super-Eddington
(SE) rates. This is consistent with Wyithe \& Loeb (2012), who found that a large fraction of galaxies at $z \gtrsim 6$ may exhibit Bondi accretion rates large enough to trap the produced photons, hence allowing for SE accretion. The authors pointed out that photon trapping can operate only for $\mathrm{BH}$ with masses $\lesssim 10^{5} \mathrm{M}_{\odot}$, as in larger holes the Bondi accretion radius exceeds the disk scale height.

Observationally, evidence for near-Eddington or SE flows has been accumulating in recent years. A study of a large sample of active galactic nuclei (AGNs) suggests that many of them emit considerably more energy and have higher Eddington ratios than previously assumed (Netzer \& Trakhtenbrot 2014). Kormendy \& Ho (2013) have argued that the normalisation of the local $\mathrm{BH}$ scaling relations should be increased by a factor of five to $M_{\mathrm{BH}}=0.5 \%$ of the bulge mass. This increases the local mass density in BHs by the same factor, decreases the required mean radiative efficiency to $1-2 \%$, and may be evidence for radiatively inefficient SE accretion (e.g. Soltan 1982; Novak 2013; see also Li 2012). At high redshifts, the very soft X-ray spectrum of ULAS $\mathrm{J} 1120+0641$ appears to suggest that this quasar is accreting at super-critical rates (Page et al. 2013). Moreover, supercritical accretion onto stellar-mass BHs has been invoked to explain the nature of the ultraluminous X-ray sources (e.g. Gladstone, Roberts, \& Done 2009; Middleton et al. 2013).

The properties of flows accreting onto BHs at SE rates were first investigated by Abramowicz et al. (1988), who found the so-called 'slim-disk solution' to the Navier-Stokes equations. The standard Shakura-Sunyaev disc solution (Shakura \& Sunayev 1973) assumes that the viscosity-generated heat is locally radiated away as a black body. It is effectively a 0th dimension model, described by algebraic equations valid at any particular radial location in the disk, independent of the flow physical conditions at larger or shorter radii. As already pointed out in the original work by Shakura \& Sunayev, at high accretion rates, i.e. when the produced luminosity is larger than $\simeq 30 \%$ the Eddington limit, the local assumption is no longer valid. Abramowicz et al. (1988) derived a more general set of non-local solutions valid in the high-accretion rate regime. The slim disk (sometimes nicknamed 'the Polish doughnut') has the form of a stationary, optically and geometrically thick accreting flow, and it is obtained by numerical integration of the two-dimensional stationary Navier-Stokes equations with a transonic point. Such solution allows for the generated heat to be advected in by the accreting gas. In simple terms, in slim disks the flow opacity is so high that the diffusion timescale of photons exceeds the accretion time scale. Part of the radiation produced is then 'trapped' within the accreting flow, and fated to feed the BH (i.e. to add to its mass). The reduced radiative efficiency opens the possibility of having extremely high mass accretion rates unimpeded by radiation, well above the Eddington limit.

Among the most advanced studies to date of the Polish doughnut are those by Sadowski and collaborators (see, e.g. Sadowski 2009, Sadowski et al. 2014), who performed MHD general relativistic axisymmetric simulations of SE accretion 

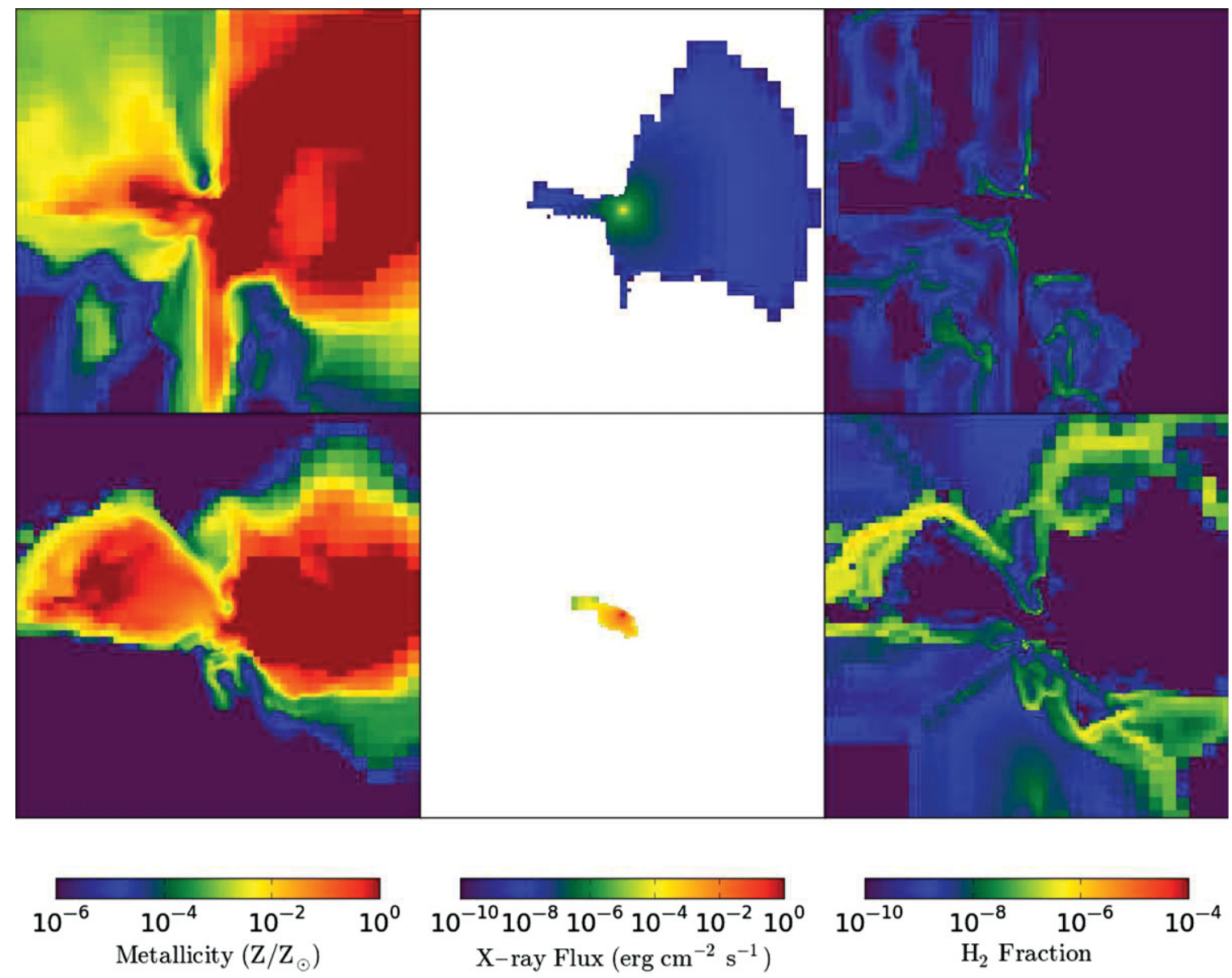

Figure 6. The metallicity (left), the X-ray flux (middle), and the $\mathrm{H}_{2}$ fraction (right) within a $1 \mathrm{kpc}$ region surrounding a central $5 \times 10^{4}$ $\mathrm{M}_{\odot}$ accreting DCBH. With a background LW radiation field of $J_{21}=10^{3}$ (bottom panels), the gas is able to cool and collapse more readily than with $J_{21}=10^{5}$ (top panels), due to stronger cooling by $\mathrm{H}_{2}$ molecules. As a result, the $\mathrm{BH}$ accretes more rapidly in the former case, although the X-rays it emits do not propagate as widely as in the latter. From Aykutalp et al. (2014).

flows. State-of-the-art three dimensional simulations with full radiative transfer (Jiang, Stone, \& Davis 2014; McKinney et al. 2014) show how vertical advection of radiation caused by magnetic buoyancy transports energy faster than photon diffusion, allowing a significant fraction of the photons to escape from the system before being advected into the hole. In the context of early BH seed growth, the interesting properties are: (i) even for the somewhat larger (compared to, e.g. Sadowski's studies) radiative efficiencies found by Jiang et al. (2014), radiation does not halt accretion as the outflowing gas and photons leave the system through a funnel near the rotation axis (while mass is accreted in the plane of the disk), and (ii) the radiative efficiency at SE rates is almost independent upon the BH spin. As highlighted by Volonteri, Silk, \& Dubus (2015), this has the important consequence that the expected BH spin-up caused by the accreted gas would not hinder the $\mathrm{BH}$ growth through a high radiative efficiency, as shown in Figure 7.

SE accretion of stellar-sized BHs has been recently recognised as a possible alterative able to explain the very existence of billion solar mass way BHs at redshifts as high as $\simeq 7$ (Madau et al. 2014; Tal \& Natarajan 2014; Volonteri et al. 2015). Volonteri et al. (2015) made specific predictions that long-lived SE accretion occurs only in galaxies with copious low-angular momentum gas, and pointed out how the dutycycle of a BH can be as low as $\sim 0.01$, to be compared to $\sim$ unity required if accretion proceeds at sub-critical pace. Madau et al. (2014) used the numerical solutions provided by Sadowski et al. (2013) in terms of radiative efficiency vs. accretion rate, and showed that, under the assumption that a gas supply rate a few times the Eddington rate $\left(\gtrsim 0.01 M_{5}\right.$ $\mathrm{M} \odot \mathrm{yr}^{-1}$ (where $M_{5}$ is the mass of the hole in units of $10^{5}$ $\mathrm{M}_{\odot}$ ) is indeed able to reach the central $\mathrm{MBH}$ for a period of 20 Myr or so, a few episodes of SE accretion may turn a light seed holes into the population of rare bright quasars observed at $z \sim 6$ by the SDSS. The high rates required may both be determined by local physics within the accretion flow (e.g. Dotan \& Shaviv 2011) as well as by the largescale cosmological environment in which MBHs and their hosts are growing (e.g. Mayer et al. 2010). By means of a semi-analytical approach, Smole, Micic, \& Matinović (2015) found that low-mass seeds $\left(M \simeq 100 \mathrm{M}_{\odot}\right)$ accreting at 3-4 

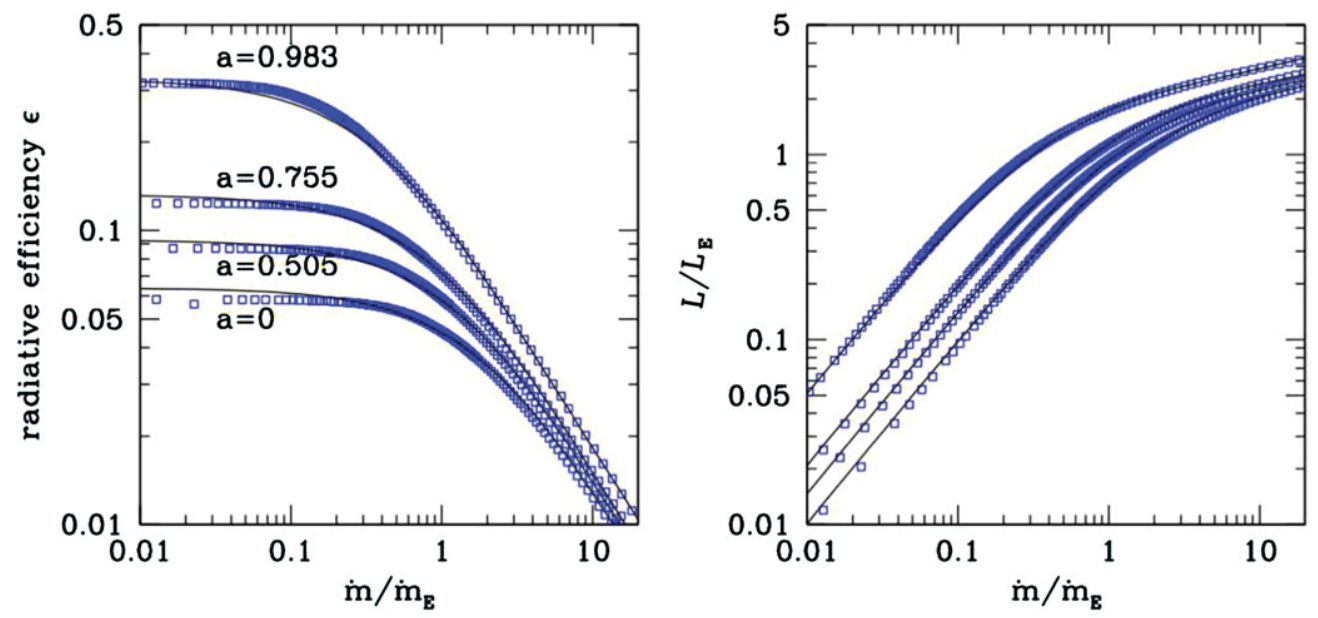

Figure 7. Radiative efficiency and total luminosity of an accreting BH are plotted in the left and right panels, respectively, as a function of the accretion rate $\dot{M}$ (in units of the Eddington rate). The blue points are the results of the numerical integration of the relativistic slim disk equations obtained by Sadowski (2009), while the solid curves from top to bottom show best-fit functions. From Madau, Haardt, \& Dotti (2014).

times the Eddington rates could in principle explain the mass function of SMBH at $z \sim 7$.

The key aspect to assess the importance of SE accretion in the early growth of BHs is the mass supply from large, kpc scales, and the ability of stellar-size BHs to intercept and accrete such inflowing gas. Two kind of approaches has been pursued so far. Small scale radiation hydrodynamical simulations, and larger scale hydro simulations in a cosmological framework.

Recently, Pacucci et al. (2015) and Inayoshi et al. (2015) elaborated upon the same concept through one dimensional and two dimensional radiation hydro-dynamical simulations of the converging flow, and found that, in certain conditions, rapid gas supply at $\mathrm{SE}$ rates from the Bondi radius can occur without being impeded by radiation feedback. In this regime, accretion is steady, and larger than 3000 times the Eddingtion rate (defined there as $\dot{m}_{\text {Edd }} \equiv L_{\text {edd }} / c^{2}$ ). As originally found by Milosavljevic et al. (2009) and by Park \& Ricotti (2011), at lower Bondi rates accretion is instead episodic, because of radiative feedback. A two dimensional analysis, performed by Yang et al. (2014), showed the importance of radiation in stabilising convection in slim disks.

Though extremely detailed, such simulations do not capture the dynamics of gas feeding of the $\mathrm{BH}$ from large scales. As an example, the three dimensional simulations by Jiang et al. (2014) cover only a small region well within the trapping radius. For such reasons, larger scale simulations of the three dimensional circum-nuclear disk have been performed. The drawback of this approach to the problem is that simulations on $\gtrsim 100 \mathrm{pc}$ scale cannot resolve the small accretion disk around the $\mathrm{BH}$, and hence one must employ 'subgrid recipes' to model the gas down to the accretion radius. Given that feedback from primordial stars and their $\mathrm{BH}$ remnants tends to strongly limit accretion (e.g. Alvarez et al. 2009), stellar-size BH seeds may not be prone to early growth (and incidentally, may not be shining as miniquasars).
The situation is likely to be quite different in more evolved galaxies, where gas is thought to form a circum-nuclear disk. In this context, very recently Lupi et al. (2016) studied the selective accretion of stellar-mass seeds in the gaseous circum-nuclear disk expected to exist in the cores of highredshift galaxies. Their sub-pc resolution hydrodynamical simulations showed that stellar-mass holes orbiting within the central $100 \mathrm{pc}$ of the circum-nuclear disk bind to very high-density gas clumps that arise from the fragmentation of the surrounding gas. Owing to the large reservoir of dense cold gas available, a stellar-mass $\mathrm{BH}$ growing at $\mathrm{SE}$ rates according to the slim disc solution can increase its mass by three orders of magnitudes within a few million years. The low radiative efficiency of super-critical accretion flows is instrumental to the rapid mass growth of the $\mathrm{BHs}$, as they imply modest radiative heating of the surrounding nuclear environment. The left panels of Figure 8 show BH masses as functions of time, while central and right panels compare the case of SE accretion with a standard case of $10 \%$ radiative efficiency. The results of Lupi et al. (2015) indicate that a short phase of SE accretion onto stellar-size BHs in the core of star-forming galaxies at $z \gtrsim 10$ may be a first step toward the formation of the supermassive BHs powering the brightest QSOs observed at $z \lesssim 7$.

\section{IMPLICATIONS FOR THE FATE OF THE FIRST MASSIVE BLACK HOLES}

In order to explain the formation of the largest SMBHs inferred to power quasars at high redshift, it is necessary to model their growth across cosmic time, starting from the smaller BH seeds that form from the collapse of the earliest stars. As the relatively small DM halos in which these seeds form merge with many other halos and end up in much more massive systems, large-scale simulations are required to track their long term evolution. In turn, given finite computational 

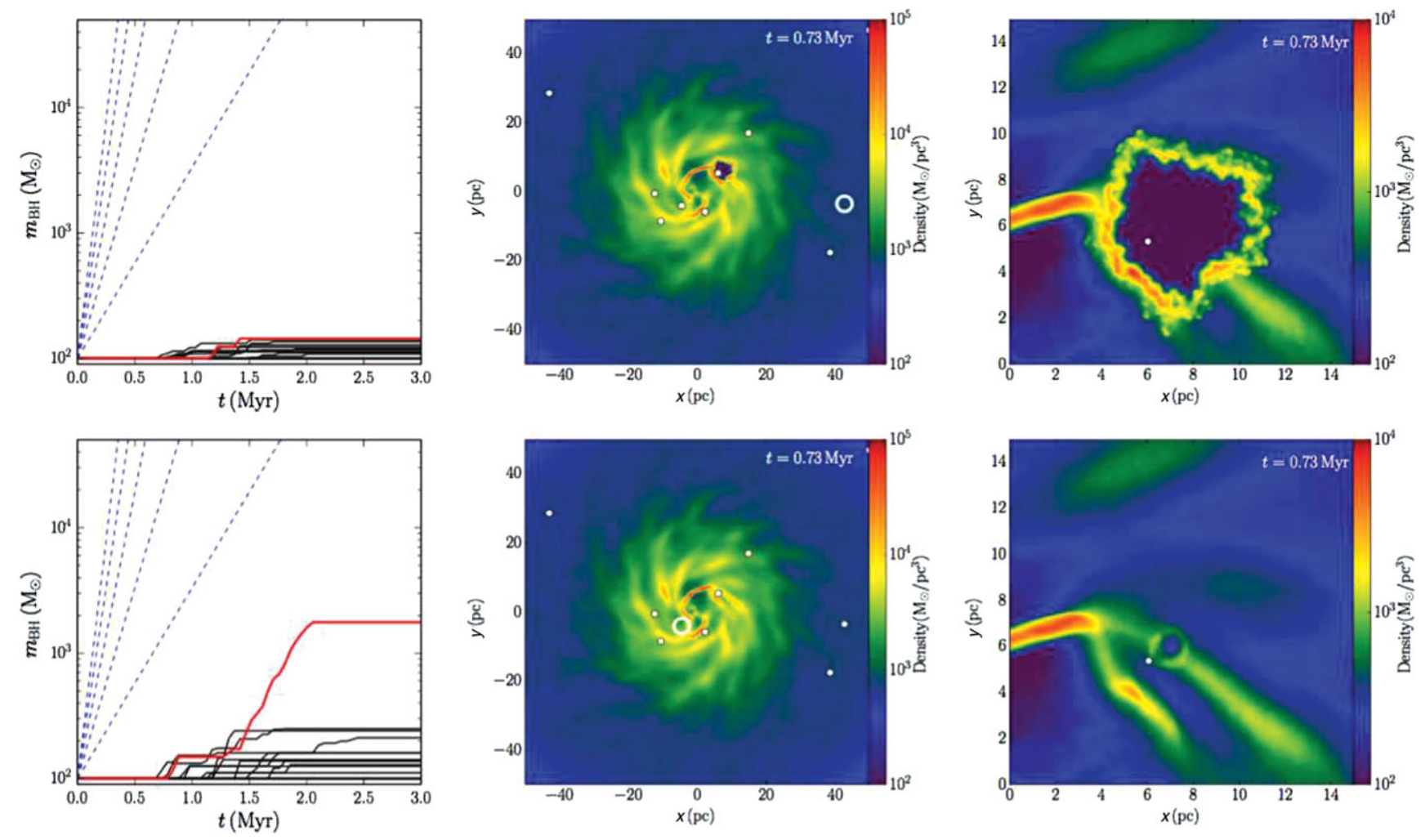

Figure 8. Left panels: BH masses as a function of time, assuming $10 \%$ radiative efficiency of accretion (top), and the radiatively inefficient slim-disk solution (bottom). The red lines correspond to the most massive BHs at the end of the runs, while the blue-dashed lines trace accretion histories at fixed Eddington ratios of 500, 400, 300, 200, and 100, respectively. Central panels: gas density maps for the two runs at $t=0.73$ Myr. Right panels: zoom in of a region heated by BH feedback. The white dots mark the positions of the BHs. From Lupi et al. (2015).

resources, such large-scale simulations generally cannot capture accretion of gas and the associated radiative feedback in great detail (e.g. Debuhr et al. 2010; Power, Nayakshin, \& King 2011; Hobbs et al. 2012; Curtis \& Sijacki 2015; RosasGuevara et al. 2015; but see also Pelupessy, Di Matteo, \& Ciardi 2007).

Nonetheless, there is some encouraging consistency between models of $\mathrm{BH}$ seed formation and the observational data on high-redshift quasars, which is strengthened by largescale cosmological simulations of the evolution linking the former to the latter. In particular, the masses of $\mathrm{BH}$ seeds which are typically adopted in large-scale, relatively lowresolution cosmological simulations (e.g. Bellovary et al. 2011; Kim et al. 2011) are often consistent with the masses expected for seeds formed from the collapse of SMSs in the direct collapse model (e.g. Ferrara et al. 2014). These simulations generally show that the growth of $\sim 10^{9} \mathrm{M}_{\odot}$ SMBHs from these seeds, fueled by accretion of low angular momentum gas (e.g. Hopkins \& Quataert 2010) delivered by cold flows (e.g. Dubois et al. 2012, 2013), could proceed fast enough to explain the highest redshift quasars (Li et al. 2007; Sijacki, Springel, \& Haehnelt 2009; Sijacki et al. 2015; Di Matteo et al. 2012), as shown in Figure 9.

Observations of BHs inhabiting more local, dwarf galaxies (e.g. Reines et al. 2011; 2014; Greene 2012; Mezcua et al. 2015) and Milky Way satellites (see e.g. van Wassenhove

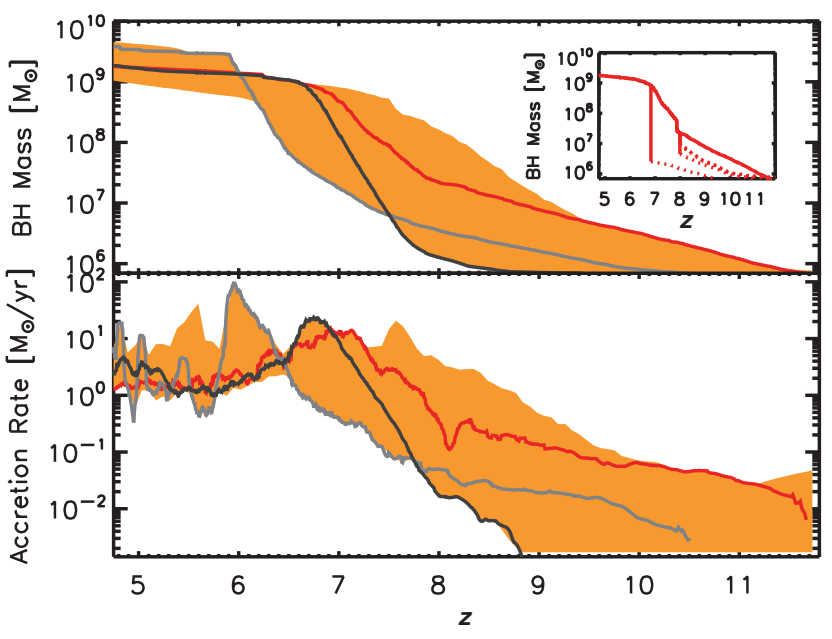

Figure 9. The accretion rate (bottom) and mass (top) of three BHs, as functions of redshift $z$, as found in a large-scale cosmological simulation. These BHs are seeded with masses consistent with DCBHs, grow to masses $\geq 10^{9} \mathrm{M}_{\odot}$ before $z=6$, and are broadly consistent with observations of quasars at these redshifts. From Di Matteo et al. (2012).

et al. 2010) may also be explained by the DCBH seed model, although it is likely that other seeding processes populate such galaxies as well. Future observations which constrain the properties of BHs in dwarf galaxies, of the BHs powering 
high redshift quasars, and of those which may reside in the brightest primordial systems, for which CR7 is a strong candidate, will be critical to determining how the first massive BHs are seeded and grow in the early universe.

\section{ACKNOWLEDGEMENTS}

The authors would like to thank Rosa Valiante, Rafaella Schneider, and Marta Volonteri for the opportunity to contribute this review. Work at LANL was done under the auspices of the National Nuclear Security Administration of the US Department of Energy at Los Alamos National Laboratory under Contract No. DE-AC5206NA25396.

\section{REFERENCES}

Abel, T., Wise, J. H., \& Bryan, G. L. 2007, ApJ, 659, L87

Abramowicz, M. A., Czerny, B., Lasota, J. P., \& Szuszkiewicz, E. 1988, ApJ, 332, 646

Agarwal, B., \& Khochfar, S. 2015, MNRAS, 446, 160

Agarwal, B., Khochfar, S., Johnson, J. L., Neistein, E., Dalla Vecchia, C., \& Livio, M. 2012, MNRAS, 425, 2854

Agarwal, B., et al. 2015, MNRAS, submitted (arXiv:1510.01733)

Alexander, D. M., \& Hickox, R. C. 2012, NewAR, 56, 93

Alvarez, M. A., Bromm, V., \& Shapiro, P. R. 2006, ApJ, 639, 621

Alvarez, M. A., Wise, J. H., \& Abel, T. 2009, ApJ, 701, 133L

Aykutalp, A., Wise, J. H., Meijerink, R., \& Spaans, M. 2014, ApJ, 797, 139

Ball, W. H., Tout, C. A., Zytkow, A. N., \& Eldridge, J. J. 2011, MNRAS, 414, 2751

Becerra, F., Greif, T. H., Springel, V., \& Hernquist, L. E. 2015, MNRAS, 446, 2380

Begelman, M. C. 2010, MNRAS, 402, 673

Begelman, M. C. 2012, ApJ, 749, L3

Begelman, M. C., Volonteri, M., \& Rees, M. J. 2006, MNRAS, 370, 289

Bellovary, J., et al. 2011, ApJ, 742, 13

Bonoli, S., Mayer, L., \& Callegari, S. 2014, MNRAS, 437, 1576

Bromm, V. 2013, RPPh, 76, 2901

Bromm, V., \& Larson, R. B. 2004, ARA\&A, 42, 79

Bromm, V., \& Loeb, A. 2003, ApJ, 596, 34

Chen, K.-J., Bromm, V., Heger, A., Jeon, M., \& Woosley, S. 2015, ApJ, 802, 13

Chen, K.-J., et al. 2014, ApJ, 790, 162

Choi, J.-H., Shlosman, I., \& Begelman, M. C. 2013, ApJ, 774, 149

Choi, J.-H., Shlosman, I., \& Begelman, M. C. 2015, MNRAS, 450, 4411

Curtis, M., \& Sijacki, D. 2015, MNRAS, 454, 3445

Davies, M. B., Miller, M. C., \& Bellovary, J. M. 2011, ApJ, 740, L42

Debuhr, J., Quataert, E., Ma, C.-P., \& Hopkins, P. 2010, MNRAS, 406, L55

Devecchi, B., \& Volonteri, M. 2009, ApJ, 694, 302

Devecchi, B., et al. 2012, MNRAS, 421, 1465

Dijkstra, M., Ferrara, A., \& Mesinger, A. 2014, MNRAS, 442, 2036

Dijkstra, M., Haiman, Z., Mesinger, A., \& Wyithe, J. S. B. 2008, MNRAS, 391, 1961

Di Matteo, T., et al. 2012, ApJ, 745, L29

PASA, 33, e007 (2016)

doi: $10.1017 /$ pasa.2016.4
Dotan, C., \& Shaviv, N. J. 2011, MNRAS, 413, 1623

Dotan, C., Rossi, E. M., \& Shaviv, N. J. 2011, MNRAS, 417, 3035

Dubois, Y., et al. 2012, MNRAS, 423, 3616

Dubois, Y., et al. 2013, MNRAS, 428, 2885

Fan, X., et al. 2006, AJ, 131, 1203

Fernandez, R., Bryan, G. L., Haiman, Z., \& Li, M. 2014, MNRAS, 439, 3798

Ferrara, A., Haardt, F., \& Salvaterra, R. 2013, MNRAS, 434, 2600

Ferrara, A., Salvadori, S., Yue, B., \& Schleicher, D. 2014, MNRAS, 443,2410

Fiacconi, D., \& Rossi, E. M. 2016, MNRAS, 455, 2

Fragos, T., et al. 2013, ApJ, 776, L31

Fryer, C. L., \& Heger, A. 2011, AN, 332, 408

Gladstone, J. C., Roberts, T. P., \& Done, C. 2009, MNRAS, 397, 1836

Glover, S. C. O. 2015, MNRAS, 453, 2901

Glover, S. C. O., \& Brand, P. W. J. L. 2003, MNRAS, 340, 210

Greif, T. H. 2015, ComAC, 2, 3

Greif, T. H., Johnson, J. L., Bromm, V., \& Klessen, R. S. 2007, ApJ, 670,1

Greene, J. E. 2012, NatCo, 3, 1304

Haiman, Z. 2013, ASSL, 396, 293

Hartwig, T., et al. 2015a, MNRAS, 452, 1233

Hartwig, T., et al. 2015b, MNRAS, submitted (arXiv:1512.01111)

Heger, A., et al. 2003, ApJ, 591, 288

Hirano, S., et al. 2014, ApJ, 781, 60

Hirano, S., et al. 2015, MNRAS, 448, 568

Hobbs, A., Power, C., Nayakshin, S., \& King, A. R. 2012, MNRAS, 421, 3443

Hopkins, P. F., \& Quataert, E. 2010, MNRAS, 407, 1529

Hosokawa, T., Omukai, K., \& Yorke, H. W. 2012, ApJ, 756, 93

Hosokawa, T., Yorke, H. W., Inayoshi, K., Omukai, K., \& Yoshida, N. 2013, ApJ, 778, 178

Inayoshi, K., \& Haiman, Z. 2014, MNRAS, 445, 1549

Inayoshi, K., Haiman, Z., \& Ostriker, J. P. 2015b, MNRAS, submitted (arXiv:1511.02116)

Inayoshi, K., \& Omukai, K. 2011, MNRAS, 416, 2748

Inayoshi, K., \& Omukai, K. 2012, MNRAS, 422, 2539

Inayoshi, K., \& Tanaka, T. L. 2015, MNRAS, 450, 4350

Inayoshi, K., Visbal, E., \& Kashiyama, K. 2015a, MNRAS, 453, 1692

Islam, R. R., Taylor, J. E., \& Silk, J. 2003, MNRAS, 340, 647

Jeon, M., Pawlik, A. H., Bromm, V., \& Milosavljević, M. 2014, MNRAS, 440, 3778

Jiang, Y.-F., Stone, J. M., \& Davis, S. W. 2014, ApJ, 796, 106

Johnson, J. L., \& Bromm, V. 2007, MNRAS, 374, 1557

Johnson, J. L., Khochfar, S., Greif, T. H., \& Durier, F. 2011, MNRAS, 410, 919

Johnson, J. L., Whalen, D. J., Fryer, C. L., \& Li, H. 2012, ApJ, 750, 66

Johnson, J. L., Whalen, D. J., Li, H., \& Holz, D. E. 2013a, ApJ, 771,116

Johnson, J. L., Whalen, D. J., Agarwal, B., Paardekooper, J.-P., \& Khochfar, S. 2014, MNRAS, 445, 686

Johnson, J. L., et al. 2013b, ApJ, 775, 107

Katz, H., Sijacki, D., \& Haehnelt, M. 2015, MNRAS, 451, 2352

Kawakatu, N., \& Wada, K. 2009, ApJ, 706, 676

Kim, J.-H., Wise, J. H., Alvarez, M. A., \& Abel, T. 2011, ApJ, 738, 54

Kitayama, T., \& Yoshida, N. 2005, ApJ, 630, 675 
Kitayama, T., Yoshida, N., Susa, H., \& Umemura, M. 2004, ApJ, 613,631

Kormendy, J., \& Ho, L. C. 2013, ARA\&A, 51, 511

Koushiappas, S. M., Bullock, J. S., \& Dekel, A. 2004, MNRAS, 354, 292

Kuhlen, M., \& Madau, P. 2005, MNRAS, 363, 1069

Latif, M. A., Niemery, J. C., \& Schleicher, D. R. G. 2014a, MNRAS, 440, 2969

Latif, M. A., Schleicher, D. R. G., \& Hartwig, T. 2015, MNRAS, submitted (arXiv:1510.02788)

Latif, M. A., Schleicher, D. R. G., Schmidt, W., \& Niemeyer, J. 2013, MNRAS, 436, 2989

Latif, M. A., \& Volonteri, M. 2015, MNRAS, 452, 1026

Latif, M. A., et al. 2014, ApJ, 792, 78L

Latif, M. A., et al. 2015, MNRAS, 446, 3163

Li, L.-X. 2012, MNRAS, 424, 1461

Li, Y., et al. 2007, ApJ, 665, 187

Lodato, G., \& Natarajan, P. 2006, MNRAS, 371, 1813

Luo, Y., Nagamine, K., \& Shlosman, I. 2015, MNRAS, submitted (arXiv:1512.03822)

Lupi, A., Colpi, M., Devecchi, B., Galanti, G., \& Volonteri, M. 2014, MNRAS, 442, 3616

Lupi, A., Haardt, F., Dotti, M., Fiacconi, D., Mayer, L., \& Madau, P. 2016, MNRAS, 456, 2993

Machacek, M. E., Bryan, G. L., \& Abel, T. 2003, MNRAS, 338, 273

Madau, P., Haardt, F., \& Dotti, M. 2014, ApJ, 784, L38

Madau, P., \& Rees, M. J. 2001, ApJ, 551, L27

Matsumoto, T., Nakauchi, D., Ioka, K., Heger, A., \& Nakamura, T. 2015, ApJ, 810, 64

Mayer, L., Kazantzidis, S., Escala, A., \& Callegari, S. 2010, Nature, 466, 1082

Mayer, L., et al. 2015, ApJ, 810, 51

McKinney, J. C., Tchekhovskoy, A., Sadowski, A., \& Narayan, R. 2014, MNRAS, 441, 3177

Mezcua, M., Civano, F., Fabbiano, G., Miyali, T., \& Marchesi, S. 2015, ApJ, 817, 20

Middleton, M. J., Miller-Jones, J. C. A., Markoff, S., et al. 2013, Nature, 493, 187

Milosavljević, M., Bromm, V., Couch, S. M., \& Oh, S. P. 2009, ApJ, 698, 766

Mirabel, I. F., et al. 2011, A\&A, 528, 149

Montero, P. J., Janka, H.-T., \& Müller, E. 2012, ApJ, 749, 37

Mortlock, D. J. 2011, Nat, 474, 616

Netzer, H., \& Trakhtenbrot, B. 2014, MNRAS, 438, 672

Novak, G. S. 2013, MNRAS, submitted

O'Shea, B. W., \& Norman, M. L. 2007, ApJ, 654, 66

Pacucci, F., \& Ferrara, A. 2015, MNRAS, 448, 104

Pacucci, F., Volonteri, M., \& Ferrara, A. 2015, MNRAS, 452, 1922

Page, M. J., Simpson, C., Mortlock, D. J., et al. 2013, MNRAS, 440, L91

Pallottini, A., et al. 2015, MNRAS, 453, 2465

Park, K.-H., \& Ricotti, M. 2011, ApJ, 739, 2

Park, K.-H., \& Ricotti, M. 2012, ApJ, 747, 9

Pelupessy, F. I., Di Matteo, T., \& Ciardi, B. 2007, ApJ, 665, 107

Petri, A., Ferrara, A., \& Salvaterra, R. 2012, MNRAS, 422, 1690

Portegies Zwart, S. F., et al. 2004, Nature, 428, 724

Power, C., Nayakshin, S., \& King, A. 2011, MNRAS, 412, 269

Prieto, J., Jimenez, R., \& Haiman, Z. 2013, MNRAS, 436, 2301

Regan, J. A., \& Haehnelt, M. G. 2009, MNRAS, 393, 858
Regan, J. A., Johansson, P. H., \& Wise, J. H. 2015, MNRAS, submitted (arXiv:1511.00696)

Reines, A. E., et al. 2011, Nature, 470, 66

Reines, A. E., et al. 2014, ApJ, 787, L30

Reisswig, C., et al. 2013, PhRvL, 111, 1101

Ritter, J. S., et al. 2012, ApJ, 761, 56

Rosas-Guevara, Y. M., et al. 2015, MNRAS, 454, 1038

Ryu, T., Tanaka, T. L., \& Perna, R. 2015, MNRAS, 456, 223-238

Sadowski, A. 2009, ApJS, 183, 171

Sadowski, A., Narayan, R., McKinney, J. C., \& Tchekhovskoy, A. 2014, MNRAS, 439, 503

Sakurai, Y. 2015a, submitted (arXiv:1511.06080)

Sakurai, Y., Hosokawa, T., Yoshida, N., \& Yorke, H. W. 2015b, MNRAS, 452, 755

Schaerer, D. 2002, A\&A, 382, 28

Schleicher, D. R. G., Palla, F., Ferrara, A., Galli, D., \& Latif, M. 2013, A\&A, 558, 59

Schneider, R., Ferrara, A., Natarajan, P., \& Omukai, K. 2002, ApJ, 571,30

Sethi, S., Haiman, Z., \& Pandey, K. 2010, ApJ, 721, 615

Shakura, N. I., \& Sunyaev, R. A. 1973, A\&A, 24, 337

Shang, C., Bryan, G. L., \& Haiman, Z. 2010, MNRAS, 402, 1249

Shibata, M., \& Shapiro, S. L. 2002, ApJ, 572, L39

Shlosman, I., Choi, J.-H., Begelman, M. C., \& Nagamine, K. 2015, MNRAS, 456, 500-511

Sijacki, D., Springel, V., \& Haehnelt, M. G. 2009, MNRAS, 400, 100

Sijacki, D., et al. 2015, MNRAS, 452, 575

Smole, M., Micic, M., \& Matinović, N. 2015, MNRAS, 451, 1964

Sobral, D., et al. 2015, ApJ, 808, 139

Soltan, A. 1982, MNRAS, 200, 115

Sugimura, K., Coppola, C. M., Omukai, K., Galli, D., \& Palla, F. 2015, MNRAS, 456, 270-277

Sugimura, K., Omukai, K., \& Inoue, A.-K. 2014, MNRAS, 445, 544

Susa, H., Hasegawa, K., \& Tominaga, N. 2014, ApJ, 792, 32

Tal, A., \& Natarajan, P. 2014, Science, 345, 1330

Tanaka, T. L., \& Li, M. 2014, MNRAS, 439, 1092

Van Borm, C., et al. 2014, A\&A, 572, 22

van Wassenhove, S., Volonteri, M., Walker, M. G., \& Gair, J. R. 2010, MNRAS, 408, 1139

Vasiliev, E. O., Vorobyov, E. I., \& Shchekinov, Y. A. 2008, A\&A, 489,505

Venemans, B. P., et al. 2013, ApJ, 779, 24

Visbal, E., Haiman, Z., \& Bryan, G. L. 2014a, MNRAS, 442, L100

Visbal, E., Haiman, Z., \& Bryan, G. L. 2014b, MNRAS, 445, 1056

Volonteri, M. 2010, ARA\&A, 18, 279

Volonteri, M. 2012, Science, 337, 544

Volonteri, M., \& Bellovary, J. 2012, RPPh, 75, 14901

Volonteri, M., \& Rees, M. J. 2005, ApJ, 633, 624

Volonteri, M., Silk, J., \& Dubus, G. 2015, ApJ, 804, 148

Whalen, D. J., Abel, T., \& Norman, M. L. 2004, ApJ, 610, 14

Whalen, D. J., \& Fryer, C. L. 2012, ApJ, 756, L19

Whalen, D. J., van Veelen, B., O'Shea, B. W., \& Norman, M. L. 2008, ApJ, 682, 49

Whalen, D. J., et al. 2013a, ApJ, 774, 64

Whalen, D. J., et al. 2013b, ApJ, 777, 99

Whalen, D. J., et al. 2013c, ApJ, 778, 17

Willott, C. J., et al. 2003, ApJ, 587, L15

Wise, J. H., \& Abel, T. 2008, ApJ, 685, 40

Wise, J. H., Turk, M. J., \& Abel, T. 2008, ApJ, 682, 745 
Wolcott-Green, J., Haiman, Z., \& Bryan, G. L. 2011, MNRAS, 418, 838

Wu, X.-B., et al. 2015, Nature, 518, 512

Wyithe, J. S. B., \& Loeb, A. 2012, MNRAS, 425, 2892

Xu, H., Wise, J. H., \& Norman, M. L. 2013, ApJ, 773, 83

Xu, H., et al. 2014, ApJ, 791, 110
Yajima, H., \& Khochfar, S. 2016, MNRAS, 457, 2423

Yang, X.-H., Yuan, F., Ohsuga, K., \& Bu, D.-F. 2014, ApJ, 780, 79

Yoshida, N., Omukai, K., \& Hernquist, L. 2008, Science, 321, 669

Yue, B., Ferrara, A., Salvaterra, R., Xu, Y., \& Chen, X. 2014, MNRAS, 440, 1263 